\title{
Изотерма сорбции сапонина Quillaja Saponaria Molina на полимерном сверхсшитом сорбенте MN-200
}

\author{
Мироненко Н.В. ${ }^{1}$, Смусева С.О. ${ }^{1}$, Селеменев В.Ф. ${ }^{1}$, Бородкина Т.А. ${ }^{2}$ \\ ${ }^{I}$ ФГБОУ ВО «Воронежский государственный университет», Воронеж \\ ${ }^{2}$ Россошанский филиал АНОО (ВОВЭПИ), Россошь, Воронежская область
}

Поступила в редакцию 3.05.2017 г.

Изучена сорбция сапонина из водного раствора на сверхсшитом полимерном сорбенте MN200. Проведен анализ изотермы сапонина с применением адсорбционных теорий Ленгмюра, Фрейндлиха, БЭТ. Рассчитаны равновесные параметры сорбции: максимальная емкость монослоя, константы, характеризующие взаимодействия типа сорбат-сорбент, а также типа сорбат-сорбат. Установлено, что адсорбция сапонина на указанном сорбенте описывается моделью БЭТ.

Ключевые слова: сапонин, модели, межмолекулярные взаимодействия, изотерма сорбции/

\section{Sorption isotherm of saponin Quillaja Saponaria Molina on polymer super-crosslinked sorbent $\mathrm{MN}-200$}

\author{
Mironenko N.V. ${ }^{1}$, Smuseva S.O. ${ }^{1}$, Selemenev V.F. ${ }^{1}$, Borodkina T.A. ${ }^{2}$ \\ Voronezh state Universyty, Voronezh \\ Rossoshansk branch of ANOO (VO-VEPI), Rossosh
}

Traditionally extracted from plant material fraction of natural glycosides triterpene saponins extraction method contain a mixture close to the structure of the saponins, and used solvents and process conditions may cause the destruction of their structure. Unique physical properties and well-developed porous structure of the polymer MN-200 allow you to optimize technological processes of obtaining and separation of natural compounds class of terpenes.

The aim of this work was to study equilibrium sorption of triterpene saponin Quillaja Saponaria Molina on polymer sorbent MN-200, and the analysis of the isotherm with the use of classical models.

The object of the study was a sample of the saponin Quillaja Saponaria Molina production Belgium. Styrosorb MN-200 belongs to the group of organic polymer sorbents - supersewn polymer materials based on styrene and divinylbenzene with a high surface area and macroporous structure. Sorption equilibrium in the system saponin - sorbent MN-200 was studied at a temperature of $295 \pm 2 \mathrm{~K}$ under static conditions by the method of variable concentrations.

The obtained isotherm sorption of saponin according to the classification of Giles meets 4L-type. The linear dependence of the sorption parameter and the formation of a plateau on isotherme in the region of low concentrations correspond to the formation of a monolayer of saponin on the surface of the sorbent. The formation of this inflection is caused primarily by interaction of free saponin molecules with the matrix of the sorbent.

The increase in sorption parameter once in solution, the concentration of saponin will exceed the critical concentration of micelle formation (CCM) by the association of glycoside molecules coming to the surface section of phases, existing in the adsorption layer of the associates.

For the quantitative description of sorption in the interval of concentrations used linearizable equations of the theories of Langmuir, Freundlich and BET.

The high value of the correlation coefficient, as well as the comparability of the values of the constants $\mathrm{K}_{\mathrm{L}}$ and $\mathrm{K}_{\mathrm{S}}$ with the literature data allows the use of a BET equation to describe multi-molecular sorption of saponin on the surface of the sorbent MN-200.

Keywords: Saponin, models, intermolecular interactions, sorption isotherms 


\section{Введение}

Сорбенты на основе сверхсшитого полистирола MN-200 с успехом применяются для разделения ряда классов органических соединений. Возможность использования любых жидких сред позволяет решать широкий спектр задач сорбционного выделения и концентрирования. Традиционно выделяемые из растительного материала фракции природных гликозидов-тритерпеновых сапонинов экстракционным способом [1-3] содержат смесь близких по структуре сапонинов, а применяемые при этом растворители и условия проведения процесса могут вызвать разрушение их структуры. Уникальные физические свойства и развитая пористая структура полимера MN-200 позволяют оптимизировать технологические процессы получения и разделения природных соединений класса терпенов.

Данная работа посвящена описанию равновесной сорбции тритерпенового сапонина Quillaja Saponaria Molina на сверхсшитом полимерном сорбенте MN-200 с применением моделей Ленгмюра, Фрейдлиха, БЭТ.

\section{Эксперимент}

Объектом исследования являлся образец сапонина Quillaja Saponaria Molina производства Бельгия (фирма Acrus), структурная формула которого приведена на рис. 1. Основные характеристики образца [4-5] приведены в таблице 1.

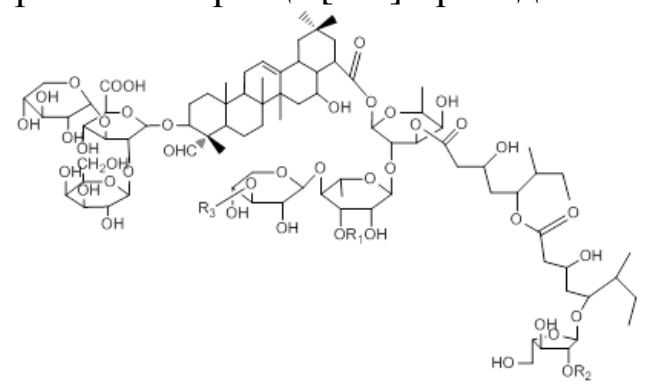

Рис. 1. Структурная формула индивидуального сапонина Quillaja Saponaria Molina $\left(\mathrm{R}_{1}-\mathrm{C}_{9} \mathrm{H}_{12} \mathrm{O}_{9}, \mathrm{R}_{2}-\mathrm{C}_{6} \mathrm{H}_{12} \mathrm{O}_{5}, \mathrm{R}_{3}-\mathrm{C}_{5} \mathrm{H}_{10} \mathrm{O}_{5}\right.$ (апиоза)).

Таблица 1. Характеристики тритерпенового сапонина Quillaja Saponaria Molina

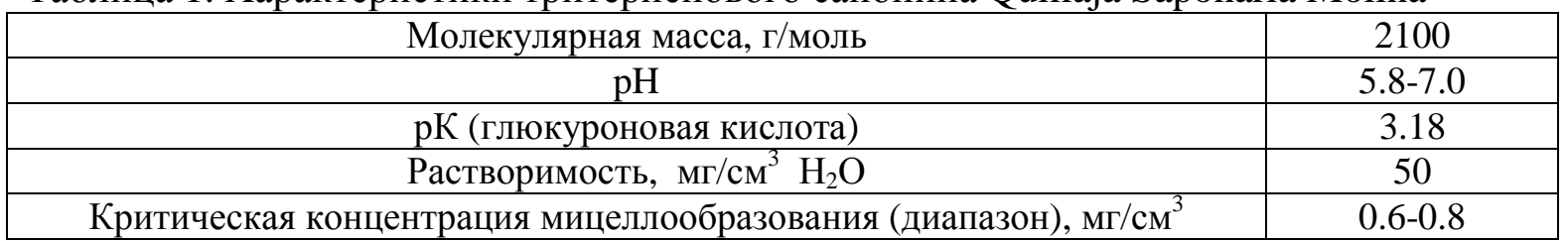

В состав углеводной цепочки сапонина входит глюкуроновая кислота, карбоксильная группа которой может находиться в растворе в зависимости от $\mathrm{pH}$ среды в молекулярной и диссоциированной форме (рис. 2).

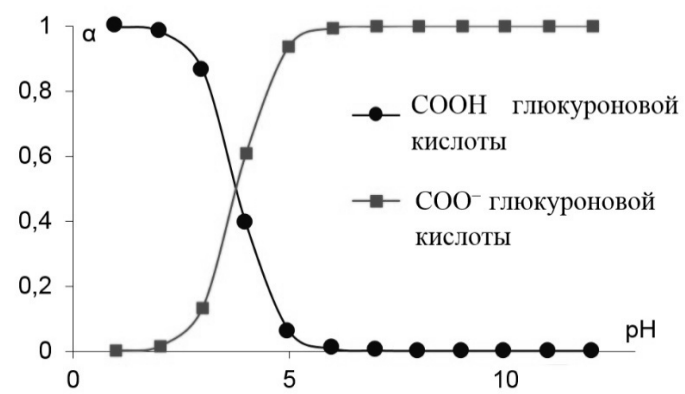

Рис. 2. Диаграмма состояния сапонина. 
Стиросорб MN-200 (рис. 3) относится к группе органических полимерных сорбентов -сверхсшитых полимерных материалов на основе стирола и дивинилбензола с высоко развитой поверхностью (от 900 до $\left.1200 \mathrm{~m}^{2} / \Gamma\right)$ и макропористой структурой.

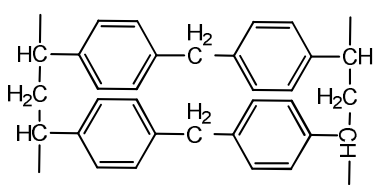

Рис. 3. Структурная формула сорбента MN-200

Основные характеристики сорбента MN-200 представлены в таблице 2.

Таблица 2. Характеристики полимерного сорбента MN-200 [6]

\begin{tabular}{|c|c|}
\hline Удельная поверхность, $\mathrm{m}^{2} / \Gamma$ & $800-1000$ \\
\hline Удельный объем, $\mathrm{cm}^{3} / \Gamma$ & $1-1.1$ \\
\hline $\mathrm{d}$ микропоры, $\AA$ & 15 \\
\hline $\mathrm{d}$ мезо и макропоры, $\AA$ & $850-950$ \\
\hline Влажность, $\%$ & $30-36$ \\
\hline
\end{tabular}

В эксперименте использовали гранулы диаметром 0.5-1 мм. Сорбционное равновесие в системе сапонин- сорбент MN-200 изучали при температуре $295 \pm 2$ K в статических условиях методом переменных концентраций. Навеску сорбента массой $1.0 \pm 0.0002$ г в воздушно-сухом состоянии заливали в конической колбе с притертой

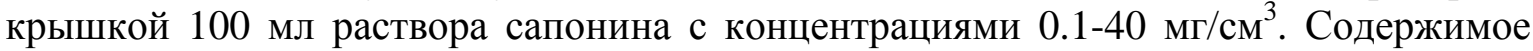
колб выдерживали при перемешивании в течение 2 часов до установления равновесия в системе. Время, необходимое для установления равновесия, определяли в предварительных кинетических экспериментах [7]. На анализ отбирали по $10.00 \mathrm{~cm}^{3}$ раствора через 2 часа от момента начала опыта при постоянной температуре $25^{\circ} \mathrm{C}$. Отобранные аликвоты анализировали на содержание сапонина спектрофотометрически по методике [8].

\section{Обсуждение результатов}

По экспериментальным данным построена изотерма сорбции сапонина (рис. 4), которая по классификации Джайлса соответствует 4L-типу. Полученная изотерма сорбции сапонина в интервале концентраций 0-5 ммоль/дм ${ }^{3}$ имеет выпуклый вид на начальном участке.Линейная зависимость величины сорбционного параметра и образование плато на изотерме в области малых концентраций отвечают формированию монослоя сапонина на поверхности сорбента.

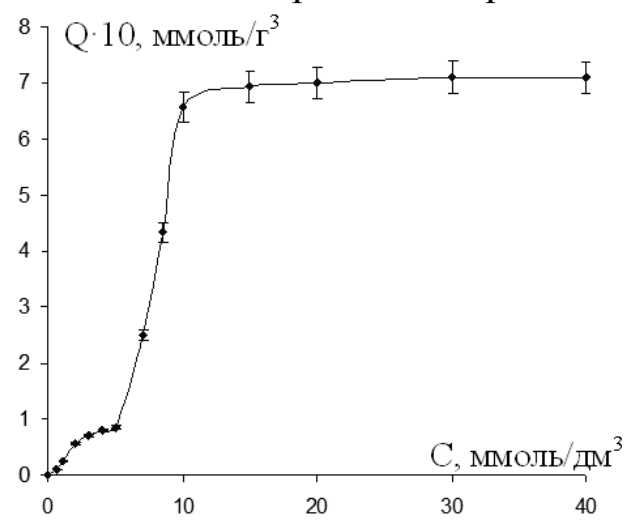

Рис. 4. Изотерма сорбции сапонина на полимерном сорбенте MN-200 при 295 К. 
Наличие этого участка является важным при выборе динамических условий сорбции. Образование данного перегиба обусловлено преимущественно взаимодействием свободных молекул сапонина с матрицей сорбента.При этом основу сорбционных процессов составляют слабые Ван-дер-Ваальсовы взаимодействия (дисперсионные силы) и водородные связи. Возможность реализации дисперсионных взаимодействий с участием гидрофобной части молекулы сапонина - агликона (олеаноловой, квиллайевой кислот) была неоднократно показана в работах [9-10]. Можно полагать, что структура данного сорбента, в частности максимальная степень сшивки, делает данный тип связи одним из доминирующих в сорбционном поглощении.

Увеличение сорбционного параметра после того, как в растворе концентрация сапонина превысит критическую концентрацию мицеллообразования (ККМ), осуществляется путем ассоциации молекул гликозида, поступающих к поверхности раздела фаз, с уже существующими в адсорбционном слое ассоциатами. Крутой рост изотермы сорбции при $\mathrm{C}_{\text {рано }}=10$ ммоль/дм ${ }^{3}$ обусловлен трехмерной ассоциацией в системе. Учитывая, что интенсивность дисперсионных сил, обуславливающих сорбцию, убывает пропорционально третьей степени расстояния молекулы от поверхности сорбента, можно заключить, что за пределами мономолекулярного слоя условия ассоциации молекул сапонина в адсорбционной фазе не отличаются от условий ассоциации в растворе. Принимая во внимание сходство условий возникновения ассоциатов в обеих фазах, можно полагать, что размеры и форма ассоциатов на поверхности сорбента в значительной мере воспроизводят структуру мицелл в равновесном растворе [11-12].

На рис. 5. представлено изменение коэффициента распределения, рассчитанного с учетом общего содержания воды в сорбенте.

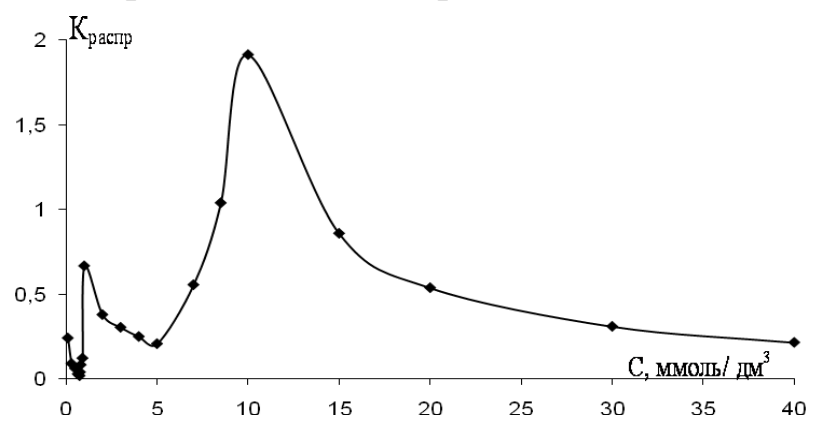

Рис. 5. Зависимость коэффициентов распределения от концентрации раствора сапонина.

В исследуемом диапазоне концентраций коэффициент распределения имеет значения ниже единицы, что свидетельствует о концентрировании вещества во

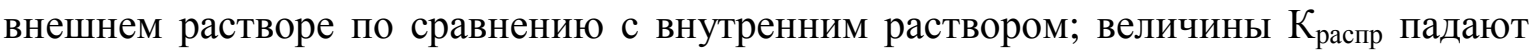
по мере выхода изотермы на плато в результате уменьшения концентрирования в сорбенте при росте концентрации внешнего раствора. Первый максимум на кривой соответствует начальному участку изотермы сорбции, на котором накопление вещества в сорбенте наиболее интенсивно. Второй максимум при $\mathrm{C}=10$ ммоль/дм ${ }^{3}$ соответствует возникновению полимолекулярного слоя адсорбированного вещества.

Для количественного описания сорбции в данном интервале концентраций использовали уравнения теорий Ленгмюра, Фрейндлиха и БЭТ.

Модель изотермы Ленгмюра основана на том, что локализация вещества, вызванная силами межмолекулярного взаимодействия, происходит на активных центрах, присутствующих на поверхности сорбента. Модель Фрейндлиха используется для описания сорбции на гетерогенной поверхности, при этом сорбционные центры

Мироненко и др. / Сорбционные и хроматографические процессы. 2017. Т. 17. № 4 
энергетически неравноценны. В основу теории полимолекулярной адсорбции БЭТ положено представление об образовании на поверхности сорбента «последовательных комплексов» адсорбционных центров с одной, двумя и т.д. молекулами сорбата.

Таблица 3. Модели, применяемые для описания изотермы сорбции сапонина

\begin{tabular}{|c|c|c|}
\hline Модель & Уравнение & Линейная форма \\
\hline Ленгмюра & $Q=\frac{Q_{\infty} K_{L} c_{p}}{\left(1+K_{L} c_{p}\right)}$ & $\frac{1}{Q}=\frac{1}{Q_{\infty} K_{L} c_{p}}+\frac{1}{Q_{\infty}}$ \\
\hline Фрейндлиха & $Q=K_{F} c_{p}^{1 / n}$ & $\ln Q=\ln K_{F}+\frac{1}{n} \ln c_{p}$ \\
\hline БЭТ & $Q=\frac{Q_{\infty} K_{L} c_{p}}{\left(1-K_{S} c_{p}\right)\left(1-K_{S} c_{p}+K_{L} c_{p}\right)}$ & $\frac{c_{p}}{Q\left(1-K_{S} c_{p}\right)}=\left(\frac{\left.K_{L}-K_{S}\right)}{Q_{\infty} K_{L}}\right) c_{p}+\frac{1}{Q_{\infty} K_{L}}$ \\
\hline
\end{tabular}

где Q-количество сорбируемого сапонина, ммоль/г; $Q_{\infty}$ - предельное количество сорбированного сапонина ммоль/г; $c_{p}$ - равновесная концентрация сапонина, ммоль/дм ${ }^{3} ; K_{L}$ - константа сорбционного равновесия, характеризующая интенсивность сорбции, дм³/ммоль; n- константа изотермы Фрейндлиха, показывающая интенсивность сорбции; $K_{F^{-}}$константа изотермы Фрейндлиха, соответствующая сорбционной емкости $\left(\text { дм }^{3}\right)^{1 / \mathrm{n}}$ (ммоль) $)^{1-1 / \mathrm{n}} / \Gamma ; K_{g}-$ константа сорбционного равновесия для полимолекулярного слоя, дм³ $/$ ммоль.

Для определения значений констант, входящих в уравнения изотерм Ленгмюра, Фрейндлиха и БЭТ, использовали линеаризованные уравнения данных моделей (табл. 3). Сорбционную емкость монослоя по уравнению Ленгмюра определяли, используя уравнение (1) как тангенс угол наклона линеаризованного уравнения, $K_{L}$ - по участку на оси ординат, отсекаемому линеаризованной прямой по уравнению (2):

$$
\begin{gathered}
Q_{\infty}=\frac{1}{k} \\
K_{L}=\frac{k}{b}
\end{gathered}
$$

Сорбционную емкость по уравнению БЭТ определяли по уравнениям (3-4):

$$
\begin{aligned}
Q_{\infty} & =\frac{1}{b \cdot K_{L}} \\
K_{L} & =\frac{k}{b}+K_{S}
\end{aligned}
$$

Параметр $K_{g}$ подбирали, исходя из максимальной величины достоверности апроксимации прямой в координатах $c_{p}-c_{p} / Q\left(1-K_{S} c_{p}\right)$.

Полученные значения параметров, входящих в уравнения моделей, а также коэффициенты корреляции приведены в таблице 4.

Таблица 4. Значения сорбционных параметров, рассчитанных с использованием уравнений Ленгмюра, Фрейндлиха, БЭТ

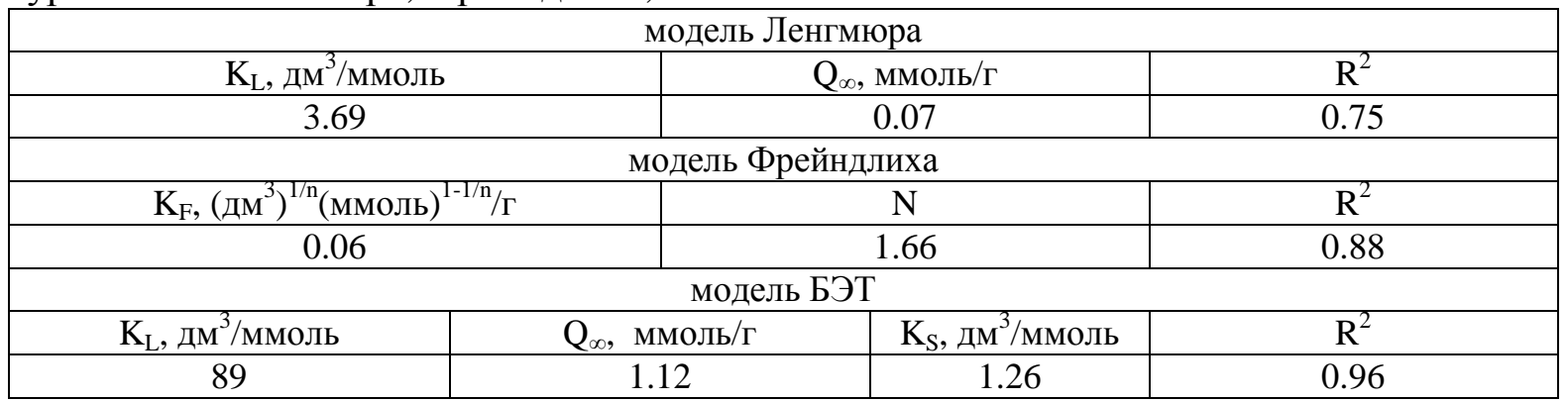


Высокое значение коэффициента корреляции, а также сопоставимость величин констант $\mathrm{K}_{\mathrm{L}}$ и $\mathrm{K}_{\mathrm{S}}$ с литературными данными [13-14], позволяет применять уравнение БЭТ для описания полимолекулярной сорбции сапонина на поверхности сорбента MN-200. Резкое нарастание слоев сорбата $\mathrm{C}=5-40$ ммоль/дм ${ }^{3}$ в фазе сорбента сопровождается усилением сорбат-сорбатных взаимодействий. Бидесмозидное строение молекулы гликозида (наличие двух углеводных остатков) позволяет модифицировать поверхность сорбента, делая ее энергетически более однородной и гидрофильной. Ассоциация как процесс структуризации и «упорядочивания» молекул сапонина реализуется в фазе сорбента в результате перегруппировок с дальнейшим изменением размера и формы мицелл.

\section{Заключение}

Уникальные физические свойства и развитая пористая структура полимеpa MN-200 позволяют значительно оптимизировать технологические процессы получения и разделения природных соединений класса терпенов.

Известно, что структура адсорбционного слоя ПАВ на твердых сорбентах является функцией химической природы сорбата и сорбента и косвенно выражается в форме изотермы сорбции. Изотерма необменной сорбции сапонина в интервале концентраций 0-1 ммоль/дм³ имеет выпуклый вид на начальном участке, далее следует точка перегиба с минимумом и ее дальнейшее возрастание.

Высокое значение коэффициента корреляции, а также сопоставимость величин констант $\mathrm{K}_{\mathrm{L}}$ и $\mathrm{K}_{\mathrm{S}}$ с литературными данными, позволяет применять уравнение БЭТ для описания полимолекулярной сорбции сапонина на поверхности сорбента MN-200.

\section{Список литературы}

1. Jiang Yu., Massiot G., Lavaud C., Teulon J.-M. et al. // Phytochemisy.1991. Vol. 30. No 7. pp. 2357-2360.

2. Лучанская Н.Н., Кондратенко Е.С., Горовиц Т.Т. // Химия природных соединений. 1971. № 2. C. 151-153.

3. Бухаров В.Т., Щербак С.П. // Химия природных соединений. 1969. № 6. С. 469-473.

4. Мироненко Н.В., Брежнева Т.А., Пояркова Т.Н., Селеменев В.Ф. // Хим.-фарм. журн. 2010. T. 44. № 3. C. 48.

5. Mitra S., Dunga S. R. // Journal Agricultuzal and Food Chemistry. 1997. № 45. P. 1587.

6. Филиппов О.А., Тихомирова Т.И., Цизин Г.И., Золотов Ю.А. // Журн. аналитической химии. 2003. Т. 58. №5. С. 454-479.

7. Селеменев В.Ф., Славинская Г.В., Хохлов В.Ю., Чикин Г.А. Практикум по ионному обмену. Воронеж: Изд-во Воронеж.ун-та. 1999. 173 с.

8. Мироненко Н.В., Брежнева Т.А., Селеменев В.Ф., Столповская А.А. // Вестник
Воронеж.гос. ун-та. Серия: Химия. Биология. Фармачия. 2015. № 1. С. 16-21.

9. Мироненко Н.В., Брежнева Т.А., Шкутина И.В., Селеменев В.Ф. // Сорбиионные и хроматографические проиессы. 2015. Т. 15. № 1. C. 74-84.

10. Мироненко Н.В., Брежнева Т.А., Селеменев В.Ф. // Журнал физической химии. 2013. Т. 87. № 3. С. 387-390.

11. Клименко Н.А., Подлеснюк В.В., Градил И.И. и др. // Коллоидный журнал. 1991. Т. 53. № 4. С.748.

12. Когановский А.М., Левченко Т.М., Кириченко В.А. Адсорбция растворенных веществ. Киев: Наукова думка. 1977. 223 с.

13. Котова Д.Л., ФамТхи Гам, Крысанова Т.А., Васильева С.Ю. // Сорбиионные и хроматографические прочессы. 2014. Т. 14. № 4. C.572-577.

14. Аскурава А.С., Синяева Л.А., Беланова Н.А., Карпов С.И. и др.//Сорбционные и хроматографические прочессы. 2016. Т. 16. № 2. C.226-233. 


\section{References}

1. Jiang Yu., Massiot G., Lavaud C., Teulon J.-M. et al. , Phytochemistri, 1991, Vol. 30, No 7, pp. 2357-2360.

2. Luchanskaja N.N., Kondratenko E.S., Gorovic T.T., Chemistry of Natural Compounds. 1971, No 2, pp. 151-153.

3. Buharov V.T., Shherbak S.P., Chemistry of Natural Compounds, 1969, No 6, pp. 469-473.

4. Mironenko N.V., Brezhneva T.A., Pojarkova T.N., Selemenev V.F., Pharmaceutical Chemistry Journal, 2010, Vol. 44, No 3, P. 48.

5. Mitra S., Dunga S.R., Journal Agricultuzal and Food Chemistry, 1997, No 45, p. 1587.

6. Filippov O.A., Tihomirova T.I., Cizin G.I., Zolotov Ju.A., Journal of Analytical Chemistry, 2003, Vol. 58, No 5, pp. 454-479.

7. Selemenev V.F., Slavinskaja G.V., Hohlov V.Ju., Chikin G.A. Praktikum po ionnomu obmenu. Voronezh: Izd-vo Voronezh.un-ta, 1999,. 173 p.

8. Mironenko N.V., Brezhneva T.A., Selemenev V.F., Stolpovskaja A.A., Vestnik Voro-

Мироненко Наталья Владимировна - к.х.н., ассистент кафедры аналитической химии Воронежского государственного университета, Воронеж, т.(473)2208-932

Смусева Светлана Олеговна - студентка химического факультета Воронежского государственного университета, Воронеж

Селеменев Владимир Федорович - д.х.н., проф., зав. каф. аналитической химии химического факультета Воронежского государственного университета, Воронеж

Бородкина Татьяна Александровна - к.б.н., директор Россошанского филиала АНОО (ВОВЭПИ), Россошь, Воронежская область nezh.gos. un-ta. Serija: Himija. Biologija. Farmacija, 2015, No 1, pp. 16-21.

9. Mironenko N.V., Brezhneva T.A., Shkutina I.V., Selemenev V.F., Sorbtsionnye $i$ khromatograficheskie protsessy,2015, Vol. 15, No 1, pp. 74-84.

10. Mironenko N.V., Brezhneva T.A., Selemenev V.F., Journal of Physical Chemistry, 2013, Vol. 87, No 3, pp. 387-390.

11. Klimenko N.A., Podlesnjuk V.V., Gradil I.I. et al., Colloid Journal, 1991, Vol. 53, No 4, pp. 748 .

12. Koganovskij A.M., Levchenko T.M., Kirichenko V.A. Adsorbcija rastvorennyh veshhestv. Kiev: Naukova dumka, 1977,223 p.

13. Kotova D.L., FamThi Gam, Krysanova T.A., Vasil'eva S.Ju., Sorbtsionnye i khromatograficheskie protsessy, 2014, Vol. 14, No.4, pp. 572577.

14. Askurava A.S., Sinjaeva L.A., Belanova N.A., Karpov S.I. et al., Sorbtsionnye i khromatograficheskie protsessy, 2016, Vol. 16, No 2, pp. 226-233.

Mironenko Natalia V. - the assistant of the department of Analitical chemistry, Voronezh State University, Voronezh, e-mail: natashamir@yandex.ru

Smuseva Svetlana O. - the student of chemical faculty, Voronezh State University, Voronezh

Selemenev Vladimir F. - the professor, head of the department of Analitical chemistry, Voronezh State University, Voronezh

Borodkina Tatyana A. - Candidate of Biological Sciences, Director of Rossoshansk branch of ANOO (VO-VEPI), Rossosh, Voronezh region 